\title{
Application of variance reduction techniques in EGSnrc based Monte-Carlo method
}

\author{
Hoang Duc Tuan ${ }^{1,2, *}$, Duong Thanh Tai ${ }^{2,3, *}$, Luong Thi Oanh ${ }^{1,2}$, Truong Thi Hong Loan ${ }^{2}$
}

${ }^{1}$ Faculty of Medicine, Nguyen Tat Thanh University, Ho Chi Minh, Vietnam

${ }^{2}$ Faculty of Physics \& Engineering Physics, University of Science, VNU-HCM, Vietnam

${ }^{3}$ Department of Radiation Oncology, Dong Nai General Hospital, Bien Hoa, Vietnam

\section{Correspondence}

Hoang Duc Tuan, Faculty of Medicine, Nguyen Tat Thanh University, Ho Chi Minh, Vietnam

Faculty of Physics \& Engineering Physics, University of Science, VNU-HCM, Vietnam

Email: hoangtuan714@gmail.com

\section{Correspondence}

Duong Thanh Tai, Faculty of Physics \& Engineering Physics, University of Science, VNU-HCM, Vietnam

Department of Radiation Oncology, Dong Nai General Hospital, Bien Hoa Vietnam

Email: thanhtai_phys@yahoo.com

\section{History}

- Received: 2018-12-06

- Accepted: 2019-05-29

- Published: 2019-06-26

\section{DOI :}

https://doi.org/10.32508/stdj.v22i2.1234

\section{Check for updates}

\section{Copyright}

(.) VNU-HCM Press. This is an openaccess article distributed under the terms of the Creative Commons Attribution 4.0 International license.

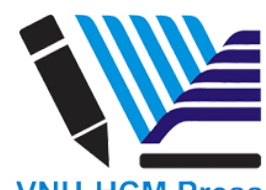

VNU-HCM Press

\begin{abstract}
Introduction: Monte Carlo (MC) is considered to be the most accurate method to calculate dose distribution in radiation therapy. However, the limitation of $\mathrm{MC}$ simulations is time-consuming to reach the desired statistical uncertainty in the dose calculation as well as in clinical practice. To overcome the disadvantages above, the variance reduction techniques (VRTs) has developed and shortened the calculation time while maintaining accuracy. Therefore, the purpose of this study is the application of VRTs in code EGSnrc to find the optimal parameters for simulation, the head of accelerator and calculation dose distribution using the MC method. Methods: The linear accelerator HPD Siemens Primus at Dong Nai General Hospital had been simulated by using BEAMnrc code and applied several VRTs such as range rejection, photon forcing, bremsstrahlung photon splitting (uniform, selective, and direction),...These VRTs were used under the same set of input parameters as histories of $2 \times 10^{8}$, the photon energy of $6 \mathrm{MV}$, structure, size and material of the phantom,...The computational efficiency $\varepsilon$ is calculated by the following equation $\varepsilon=\frac{1}{T . S^{2}}$ where Tis the CPU time of calculation and $S^{2}$ is an estimate of the variance. Then the result will be used for evaluating and selecting the VRTs, which gives the best computational efficiency. Results: The results showed a good agreement between the calculated dose and measured ones when applying different VRTs. These techniques were significantly reduced uncertainty in simulation compared the analog cases. Specifically, the efficiency of DBS and UBS improved by more than 90 times and 15 times compared with the analog instances, respectively. Rang rejection and photon forcing techniques also have improved the efficiency of simulation, but not significantly. Conclusion: The application of the VRTs for EGSnrc increase the efficiency of the simulation. VRTs is a powerful tool that should be applied for the simulation by code EGSnrc to improve calculation efficiency by reducing simulation time and its variance. Our results show that the direction bremsstrahlung splitting (DBS) gives the best computational efficiency.

Key words: Monte Carlo simulation, Variance reduction techniques, EGSnrc.
\end{abstract}

\section{INTRODUCTION}

In the simulation, the calculation time and uncertainty play an essential role in Monte Carlo simulation. It is not only directly affects the efficiency of the calculation but also affects the practicality of the application of simulation. Up to this time, there has been a lot of published research studies are demonstrating that simulation code based on Monte Carlo method can produce excellent results with reasonable uncertainties (MCNP, PENELOPE, EGS,... $)^{1-4}$. However, one of the issues associated with Monte Carlo simulations is the potentially significant $\mathrm{CPU}$ time required to reach the desired statistical uncertainty ${ }^{1,5}$ even though the strong growth of technology and the increase dramatically in the speed of computers. To resolve these issues, variance reduction techniques (VRTs) were introduced to reduce the calculation time and increase the efficiency of the simulation.
There have been numerous studies on the application of VRTs to increase the efficiency of computational simulation. In 2004, I. Kawrakow et al. studied about efficiency improvements when applying a new VRTs in BEAMnrc using directional bremsstrahlung splitting (DBS). The research concludes that the performance of DBS depends on the details of the accelerator being simulated. Increasing the field size and the photon beam at higher energies will affect the efficiency of simulation of the DBS technique ${ }^{1}$. M. Mohammeda et al. researched the VRTs available in BEAMnrc for simulation a Saturne 43 accelerator of $12 \mathrm{MV}$ photon beam. The obtained results show that employing direction bremsstrahlung photon splitting (DBS) technique alone or combined with other technologies lead to enhance the efficiency in BEAMnrc simulation $^{3}$. Most recently, S. Shanmugasundaram et al. used the VRTs to simulate various ion chambers by using EGSnrc Monte Carlo Codes. The result 
of this research determined the optimal combination of VRTs. In general, all of these above studies showed that the VRTs significantly reduce the computational time and significantly improve the efficiency of computational simulations.

EGSnrc code based on MC, it is a widely-used for simulation of radiotherapy beams. EGSnrc has two main sub-codes. The BEAMnrc is used for simulating the accelerator head, and the DOSXYZnrc is used for calculating the dose distribution ${ }^{2,6}$. This code has provided several VRTs to solve the time problem and increase the efficiency such as mentioned above, including range rejection, photon forcing, bremsstrahlung photon splitting (uniform, selective, and direction)... ${ }^{1,3,6-8}$.

The purpose of this study is to apply the VRTs of code EGSnrc for simulating and calculating the running time and the uncertainty for each simulation, from that the efficiency of each simulation model was evaluated. Then find out the VRTs will bring the highest efficiency in simulation by the MC method.

\section{MATERIAL AND METHOD}

\section{Variance reduction techniques in EGSnrc}

The variance reduction techniques are a statistical technique to simplify the calculating, reducing the time, variance, and improving the precision simulation ${ }^{1,3}$. EGSnrc applied some of the following variance reduction techniques:

\section{Range rejection}

Range rejection is a technique used to save computing time of the simulations. The basic method based on calculate the range of a charged particle and terminate its history ${ }^{6,9}$. The particle's history is terminated whenever its residual range is so low that it cannot escape from the current region or reach the region of interest ${ }^{1}$. In general, this technique is always activated in all cases of simulation with a different energy threshold (ESAVE). Following the recommendation of S. Bagheri, the value ESAVE was set to $2 \mathrm{MeV}$ in all component modules ${ }^{1,6}$. In addition, the electron and photon cutoff energies were set to ECUT $=0.70 \mathrm{MeV}$ and PCUT $=0.01 \mathrm{MeV}$, total energy included the electron rest mass energy for the charged particle ${ }^{6,10}$.

\section{Photon forcing}

This technique is useful for improving the probability of interaction of a photon with component modules of the accelerator, especially CMs is a thin slab or created from the low-density materials ${ }^{3,6,9}$. A photon forced to interact is split into a scattered photon whose weight is equal to the probability of interaction and an un-scattered photon carrying the remaining weight ${ }^{2,5,6}$.If the parent particle has not been forced to interact NFMAX times yet, these parameters will be pass onto secondary photons, and secondary photon had to interact the remaining number of times ${ }^{1,6}$.

\section{Bremsstrahlung Photon Splitting}

The main techniques of bremsstrahlung photon splitting included uniform bremsstrahlung splitting (UBS), selective bremsstrahlung splitting (SBS), and directional bremsstrahlung splitting (DBS). However, the SBS had been removed in the latest version of EGSnrc ${ }^{6}$. Therefore, we just applied UBS and DBS techniques in this work.

- Uniform bremsstrahlung splitting (UBS)

UBS had been attached in the original version of EGS ${ }^{6,9}$. When applying UBS, each bremsstrahlung produces a number of bremsstrahlung photons (NBRSPL). Each of them has a weight of being equal to the inverse of the splitting number $\frac{1}{N B R S P L}$, the weight of the electron that underwent the bremsstrahlung event ${ }^{1,3}$. The energies and directions of each photon are sampled individually according to the relevant probability distributions ${ }^{6}$. NBRSPL is a constant value set by user-input (range from 20 to 100$)^{6,11}$. The limitation of UBS is investing much of the CPU time to spent tracking split photons that will not direct to the field of interest ${ }^{1,4}$.

- Directional bremsstrahlung splitting (DBS)

DBS was introduced into EGSnrc in 2004 by Kawarkow et al. ${ }^{6}$. DBS uses a combination of interaction splitting for bremsstrahlung, annihilation, compton scattering, pair production, photoabsorption, and russian roulette to achieve much better efficiency of photon beam ${ }^{1,6}$. When using UBS, NBRSPL is defined together with the field radius (FS) of interest. The value of FS must at least encompass the entire treatment field ${ }^{2,6}$.Example, with the field size, is $10 \times 10 \mathrm{~cm}^{2}$, the value of FS should be set is $10 \mathrm{~cm}$. DBS parameters depend on the geometrical accelerator and energy of the photon ${ }^{6,9}$.

\section{Set up VRTs evaluation simulation system}

The HPD of linear accelerator Siemens Primus at the Dong Nai General Hospital was simulated by BEAMnrc. The component modules (CMs) of the accelerator include vacuum envelope (SLABS), the target (SLABS), the flattening filter (FLATFILT), ion chambers (CHAMBER), mirror (MIRROR), the jaws X and 


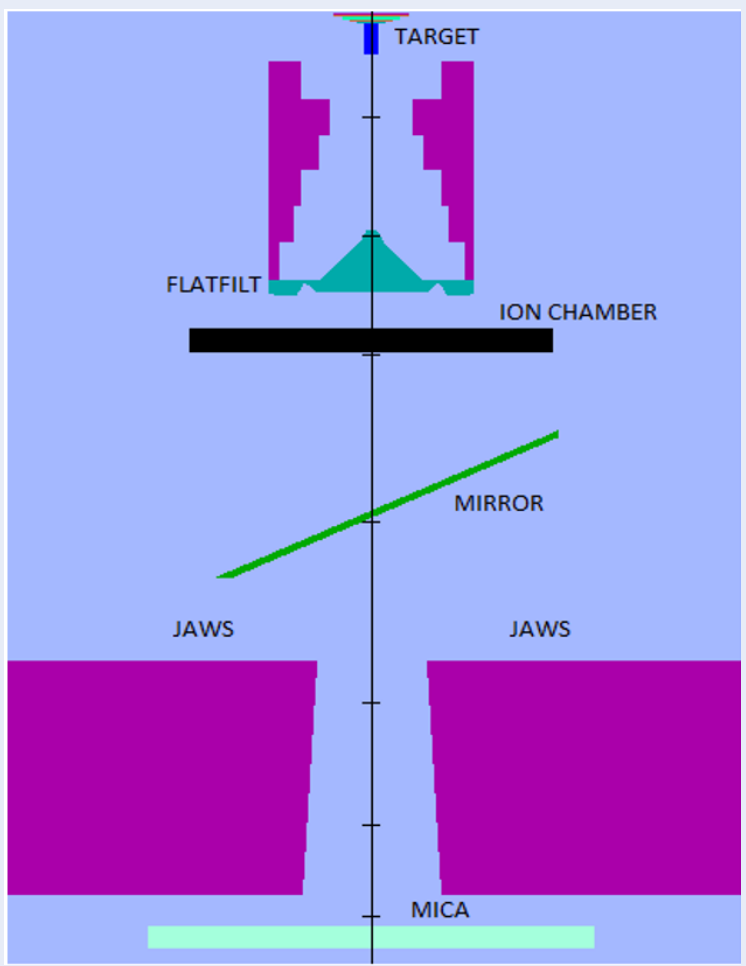

Figure 1: The component of an accelerator. Main components of the accelerator include target, flattening filter, ion chamber, mirror, Jaws $\mathrm{X}$ and Jaws $\mathrm{Y}$, and mica.

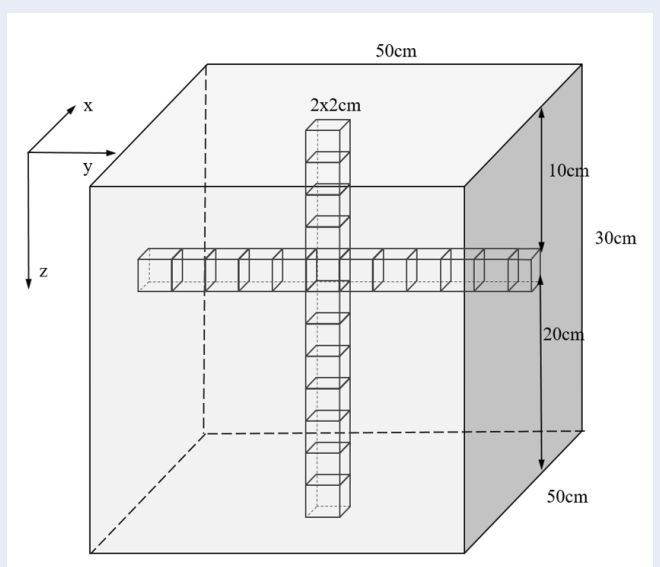

Figure 2: Do the voxels of interest in phantom. Materials of the phantom are water, dimensions of $X, Y, Z$ directions are a similarity is $50 \mathrm{~cm}$, voxels are divided into $2 \times 2 \times 2 \mathrm{~cm}^{3}$ at the center-axis and at the depth $10 \mathrm{~cm}$. 
jaws Y (JAWS), reticle tray of mica (SLABS), and slab air (SLABS). The structure of the accelerator is shown in Figure 1.

These VRTs are independently simulated with the same input parameters such as the number of histories is $5 \times 10^{8}$, the energy of photon beam is $6 \mathrm{MV}$, field sizes are $10 \times 10 \mathrm{~cm}^{2}$, distance from the source to surface distance (SSD) is $100 \mathrm{~cm}$. Besides, the structure and materials of the phantom are similar. The dose region interest of phantom at the center-axis and dose distribution at the depth of $10 \mathrm{~cm}$, voxels are divided into $2 \times 2 \times 2 \mathrm{~cm} 3$, that is present in Figure 2 .

\section{Calculation of the efficiency}

The efficiency $\varepsilon$ of a Monte Carlo simulation by the following equation:

$$
\varepsilon=\frac{1}{T \cdot S^{2}}
$$

Where $\mathrm{T}$ is the CPU time of calculation, $\mathrm{S}$ is an estimation of the uncertainty on the quantity of interest. Following the recommendation of Rogers and Mohan, an "average" uncertainty as a measure of the overall uncertainty of an MC dose calculation ${ }^{1}$, by the formula:

$$
S^{2}=\frac{1}{n} \sum_{i-1}^{n}\left(\frac{\Delta D_{i}}{D_{i}}\right)_{50 \%}^{2}
$$

Where $\mathrm{D}_{i}$ is the dose in the $\mathrm{i}$ voxel and $\Delta \mathrm{D}_{i}$ is the corresponding statistical uncertainty. To reduce the statistical uncertainty, only voxels with a dose higher than $50 \%$ of the maximum dose are accounted for in the calculation of this average quantity.

\section{RESULTS}

\section{Comparison of VRTs simulation for 6 MV photon beam.}

Firstly, each technique has been individually simulated with the number of $1 \times 10^{6}$ histories. The uncertainty was estimated on the absorbed dose at $10 \mathrm{~cm}$. The comparison of the efficiency of application variance reduction techniques in the simulation is shown in Table 1.

From Table 1, we can see that the efficiency of each simulation of VRTs has a different value. The efficiency of the DBS technique improves more 90 times compared with the analog cases. UBS techniques also have improved the efficiency, however, value the efficiency smaller than DBS about 15 times, this is because of the time simulation of UBS much too long. Rang rejection and photon forcing techniques also have improved the efficiency of simulation, but not significantly.
To increase the accuracy of the simulation and reduce error statistics. In the next step, we increased the number of histories to identify its impact on simulation efficiency. Our results are summarized in the following Table 2.

Through Table 2, with an increasing number of histories up to $5 \times 10^{8}$, we noticed that the difference rate the efficiency of various techniques is similar to the results in Table 1. DBS variance reduction technique has the highest efficiency. However, the efficiency statistical of simulation of the DBS technique increased significantly compared with those other VRTs or without VRTs. This technique improves nearly 100 times compared with the value efficiency of analog, about 83 times comparing with range rejection, 50 times and 13 times when compared with photon forcing and UBS technique, respectively. This result is in a good agreement with previous studies of Kawrakow et al. ${ }^{1,9}$.

The correlation between simulation time and its uncertainty is presented in Figure 3.

From Figure 3 obviously, UBS and DBS are techniques which significantly reduces the statistical uncertainty. Meanwhile, the reduction of statistical uncertainty of range rejection and photon forcing technique is negligible compared with the analog case.

\section{Optimization of value NBRPS for DBS}

DBS variance reduction technique has the highest efficiency. We were simulated DBS with a change of bremsstrahlung photons number (NBRPS), to find out the optimum of NBRPS value for the simulation of the $6 \mathrm{MV}$ photon beams. The results of simulation and of the efficiency are presented in Table 3.

From Table 3, the range of number bremsstrahlung photons was set from 200 to 1500 . All of these cases used DBS with different of NBRPS give more effective simulations than other VRTs (Range rejection, Photon forcing, UBS). But DBS technique with NBRPS of 1000 gives the highest efficiency. Therefore, the optimal value of NBRPS is 1000 using Directional bremsstrahlung splitting for $6 \mathrm{MV}$ photon beam simulation. It is also found in the previous researches $1,3,6$.

\section{DISCUSSION}

All of these variance reduction techniques have effective for calculating of simulation, significantly reduce uncertainty in simulation compared the analog cases. A review of results from the variance reduction experiments indicates that the amount of variance reduction by the techniques applied will vary substantially if we increase the number of histories in the simulation. 
Table 1: Comparison of variance reduction techniques simulation for $6 \mathrm{MV}$ photon beam

\begin{tabular}{lllll}
\hline Type & N of histories & CPU time $(s)$ & S & $\varepsilon$ \\
Analogue & $1 \times 10^{6}$ & 3279.1 & 0.0725 & 0.0580 \\
Range rejection & $1 \times 10^{6}$ & 2837.9 & 0.0690 & 0.0740 \\
Photon forcing & $1 \times 10^{6}$ & 1925.4 & 0.0753 & 0.0916 \\
UBS & $1 \times 10^{6}$ & 26680.6 & 0.0121 & 0.2560 \\
DBS & $1 \times 10^{6}$ & 8980.7 & 0.0046 & 5.2623 \\
\hline
\end{tabular}

Table 2: The effect of histories number on VRTs simulation

\begin{tabular}{lllll}
\hline Type & $\mathbf{N}$ of histories & CPU time $(\mathbf{s})$ & $\mathbf{S}$ & $\varepsilon$ \\
Analogue & $5 \times 10^{8}$ & 26951.2 & 0.0224 & 0.074 \\
Range rejection & $5 \times 10^{8}$ & 23108.1 & 0.0222 & 0.088 \\
Photon forcing & $5 \times 10^{8}$ & 15439.5 & 0.0211 & 0.145 \\
UBS & $5 \times 10^{8}$ & 100566.5 & 0.0040 & 0.609 \\
DBS & $5 \times 10^{8}$ & 11187.5 & 0.0035 & 7.297 \\
\hline
\end{tabular}

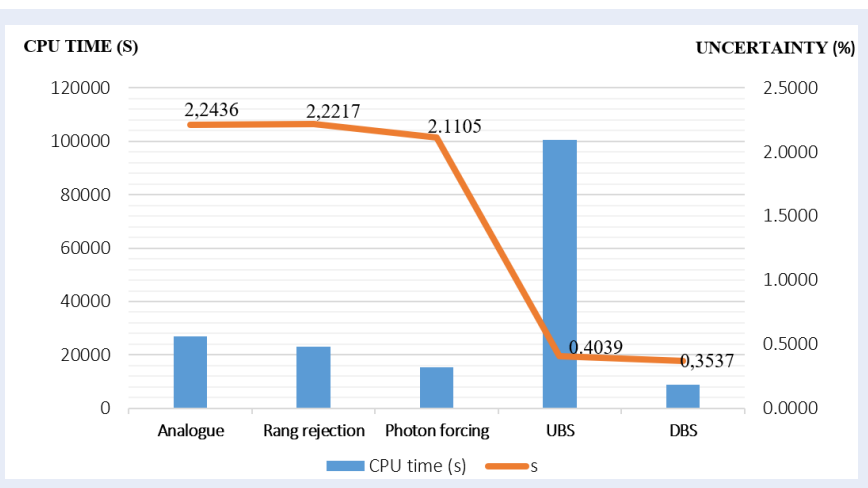

Figure 3: The correlation between time simulation and uncertainty. Simulation with the analog case or applying VRTs such as Rang rejection and Photon, forcing take a short calculation time, but the uncertainty value is quite large. Meanwhile, UBD and DBS are effective in significantly reducing the uncertainty value of the simulation process.

Table 3: The efficiency of simulation with directional bremsstrahlung splitting

\begin{tabular}{lllll}
\hline NBRPS & N of histories & CPU time $(\mathbf{s e c})$ & S & $\varepsilon$ \\
200 & $5 \times 108$ & 11763.8 & 0.0060 & 2.341 \\
500 & $5 \times 108$ & 8376.1 & 0.0049 & 4.941 \\
750 & $5 \times 108$ & 11187.5 & 0.0035 & 7.297 \\
10000 & $5 \times 108$ & 10162.8 & 0.0034 & 7.972 \\
15000 & $5 \times 108$ & 9822.6 & 0.0042 & 5.771 \\
\hline
\end{tabular}


It is demonstrated that a number of histories have a large effect on the efficiency of simulation ${ }^{3}$.

Besides, the obtained results also show that DBS and UBS are techniques that dramatically reduce the statistical uncertainty, however, due to simulation time of UBS too long compared to the DBS technique, so this technique does not bring high efficiency. Highest efficiency obtained when the DBS technique has been applied that is quite consistent with the results of previous studies ${ }^{1,3,6}$. It has been observed that the EGSnrc simulation with VRTs brings higher efficiency, reduces calculation time and minimal statistical uncertainty comparing with the ones without VRTs.

\section{CONCLUSION}

As a result, the application of the variance reduction technique for EGSnrc increased the efficiency of the simulation. We conclude that VRTs are powerful tools for improving computational efficiency. Among the specific variance reduction techniques (Range rejection, Photon forcing, UBS and DBS), DBS was found to be most effective for all the application within this study. This technique should be applied for the simulation by code EGSnrc.

\section{COMPETING INTERESTS}

The authors declare that there is no conflict of interest regarding the publication of this paper.

\section{AUTHORS' CONTRIBUTIONS}

All the authors contributed equally to the paper including the research idea, data analysis, and writing manuscript.

\section{REFERENCES}

1. Kawrakow I, Rogers D, Walters B. Large efficiency improvements in BEAMnrc using directional bremsstrahlung splitting, Medical Physics; 2004.

2. Kawrakow I. Accurate condensed history Monte Carlo simulation of electron transport. I. EGSnrc, the new EGS4 version, Medical Physics; 2000.

3. Mohammeda $M$, Chakir E, Boukhal $H$, Saeed $M$, Bardouni T. Evaluation of variance reduction techniques in BEAMnrc Monte Carlo simulation to improve the computing efficiency, Journal of Radiation Research and Applied Sciences; 2015.

4. Shanmugasundaram S, Chandrasekaran S. Optimization of variance reduction techniques used in EGSnrc Monte Carlo Codes, Journal of Medical Physics; 2018.

5. Bagheri D, Kawrakow I, Walters B, Rogers D. Monte Carlo Simulations: Efficiency Improvement Techniques and Statistica Consideration, Medical Physics; 2006.

6. Rogers D, Walters B, Kawrakow I. BEAMnrc User's Manual, National Research Council of Canada Report, Ottawa: NRC; 2005.

7. Gualdrini G, Ferrari M. Monte Carlo Variance reduction techniques: An overview with some pratical examples, Radiation Protection Dosimetry; 2011.

8. Rodriguez M, Sempau J, Brualla L. A combined approach of variance-reduction techniques for the efficient Monte Carlo simulation of linacs, Medical Physics; 2012.

9. Kawrakow I, Fippel M. Investigation of variance reduction techniques for Monte Carlo photon dose calculation using XVMC, Physics in Medicine \& Biology; 2000.

10. Fragoso M, Kawrakow I, Faddegon B, Solberg T, Chetty I. Fast, accurate photon beam accelerator modeling using BEAMnrc: A systematic investigation of efficiency enhancing methods and cross-section data, Medical Physics; 2009.

11. Kawrakow I, Rogers D. The EGSnrc code system: Monte Carlo simulation of electron and photon transport, National Research Council of Canada, Ottawa; 2003. 